\title{
Floating Dislocated Elbow: A Variant with Articular Fracture of the Humerus
}

\author{
Pablo De Carli, MD, Jorge G. Boretto, MD, Walter O. Bourgeois, MD, and Gerardo L. Gallucci, MD
}

$\mathbf{F}$ loating elbow, defined as an ipsilateral humeral and forearm fractures, is an uncommon injury. ${ }^{1-6}$ Moreover, floating dislocated elbow is an extremely rare lesion. ${ }^{7,8}$ Three cases have been reported in the english literature. ${ }^{3,7,8}$

We report a complex pattern of injury, which includes an intercondylar humeral fracture, radius and ulna shaft fractures, and dislocation of the elbow. This is a variant of floating dislocated elbow with humeral articular disruption, which has not been previously reported.

\section{CASE REPORT}

A 25-year-old woman was attended at the emergency room after suffering a car accident. Physical examination showed a markedly swollen upper dominant extremity. Deformities were present at the elbow and forearm. No neurovascular compromise was noted.

After clinical and radiographic examination a Gustilo 3A intercondylar humeral fracture was diagnosed with bone loss at the lateral column of the supracondylar aspect of the humerus (Fig. 1, A and B). The elbow showed posterior dislocation. The radius sustained an oblique Gustilo 2 fracture, across the union of the middle and distal third. The ulna suffered a segmented shaft fracture (Fig. 1C). Immediate surgical debridement was performed. After reduction of the distal humeral articular surface, a minimal temporary internal fixation was used due to the soft tissue damage. Two screws and a tension band wiring provided humeral continuity and articular congruence allowing the reduction of the elbow (Fig. 2A). A local skin flap was done to cover the posterior aspect of the elbow. Open reduction and definitive internal fixation with $3.5 \mathrm{~mm}$ dynamic compression plates (Synthes, Oberdorf, Switzerland), was performed in the radius and the ulna (Fig. 2, B and C). The upper limb was immobilized in a

Submitted for publication February 23, 2004

Accepted for publication January 13, 2005

Copyright $(9) 2006$ by Lippincott Williams \& Wilkins, Inc.

From the Hand and Upper Extremity Surgery Department, Institute of Orthopedics and Traumatology "Prof. Dr. Carlos Ottolenghi," Hospital Italiano de Buenos Aires, Ciudad Autónoma de Buenos Aires, Argentina.

Address for reprints: Jorge G. Boretto, Hospital Italiano de Buenos Aires, Potosi 4215. Ciudad Autónoma de Buenos Aires, Argentina; email: jboretto@hotmail.com.

DOI: $10.1097 / 01 . t a .0000203569 .57055 .90$

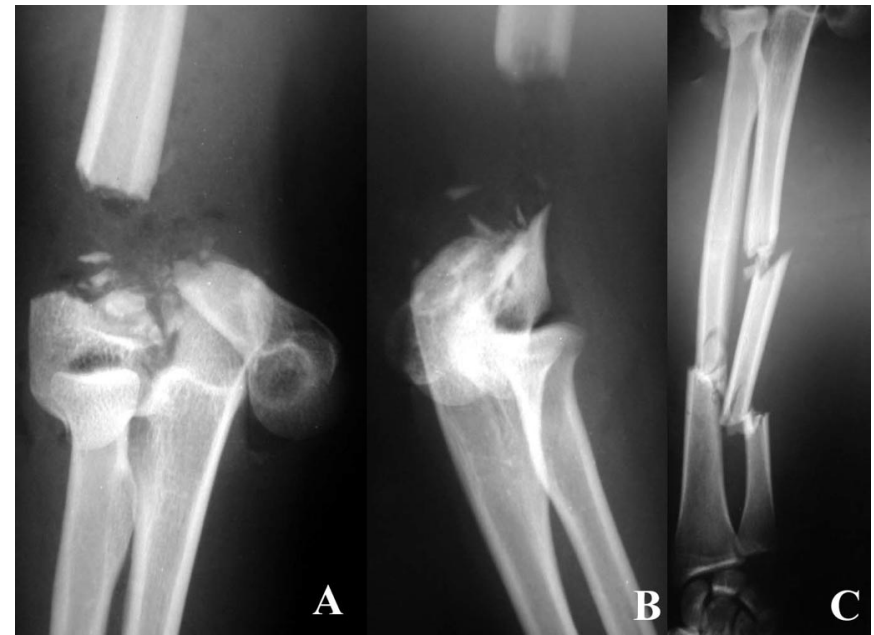

Fig. 1. Anteroposterior $(A)$ and lateral $(B)$ radiographs showing intercondylar humeral fracture. (C) Anteroposterior radiography demonstrating forearm fractures.

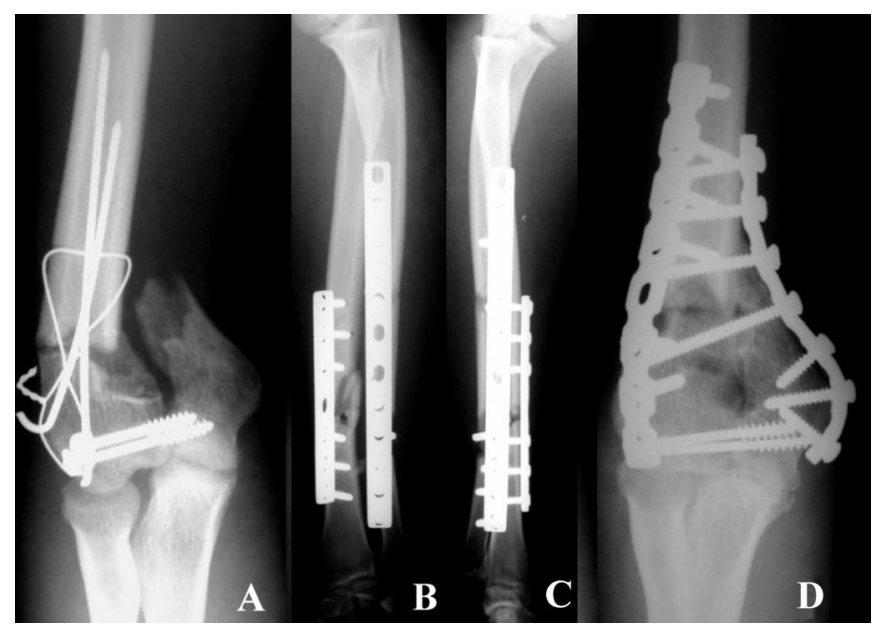

Fig. 2. (A) Minimal temporary internal fixation of the distal humerus. ( $B$ and $C$ ) Plain radiographs of the forearm showing definitive rigid internal fixation. (D) Bone graft and definitive internal fixation of the distal humerus.

posterior splint, and clinical control was done every 2 days to rule out infection.

Two weeks later, the tension band wiring was removed and an autogenous corticocancellous bone graft from the iliac 


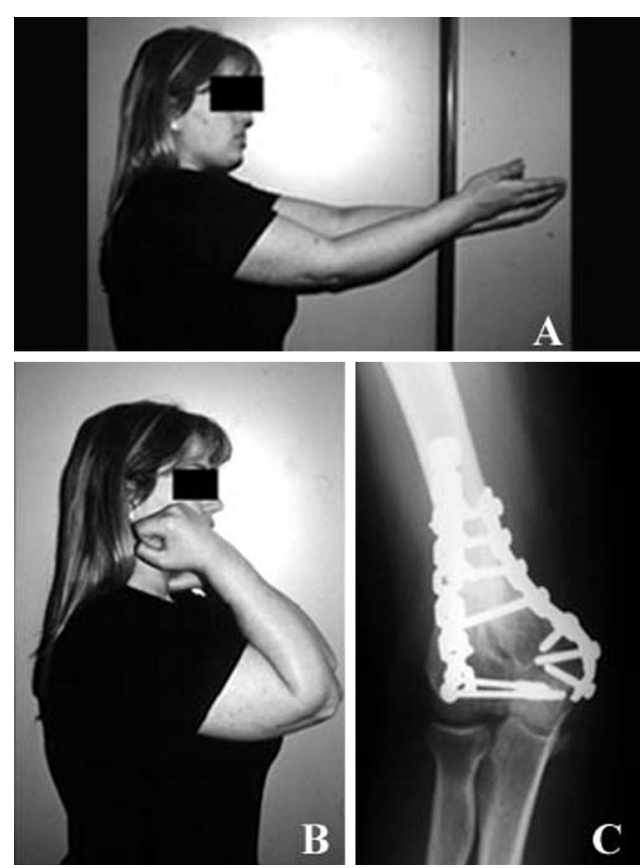

Fig. 3. (A and B) Elbow range of motion. (C) Anteroposterior radiography showing bone graft integration and fractures healed of the distal humerus.

crest was obtained to reconstruct the lateral column of the distal humerus. The definitive internal fixation was obtained with two 3.5-mm reconstruction plates (Synthes, Oberdorf, Switzerland) (Fig. 2D). The postoperative period was uneventful and active mobilization was allowed after 2 weeks.

At 2 years of follow-up, the range of motion of the elbow is 130 degrees of flexion and 45 degrees of extension (Fig. 3, A and B). Full extension could not be recovered due to the narrowing of the olecranon groove by the bone graft. Pronation and supination are complete. The fractures are healed and the bone graft is integrated (Fig. 3C). The patient is satisfied with the functional result and refuses to undergo surgical treatment to restore elbow extension.

\section{DISCUSSION}

Floating dislocated elbow faces up to the orthopedic surgeon to an extremely rare and severe injury with potentially major complications. $3,4,7,8$

Three cases have been previously reported in the English literature. Both Rogers et al. ${ }^{3}$ and Viegas et al. ${ }^{8}$ presented humeral shaft fractures, forearm fractures, and posterior elbow dislocation. The third case, reported by Sarup and Bryant, ${ }^{7}$ presented a humeral shaft fracture associated to a Galeazzi fracture and posterior elbow dislocation.

We present a not reported variant of floating dislocated elbow, which is more complex than the previously presented due to the severe injury in the articular component of the distal aspect of the humerus.

Viegas $^{8}$ described in detail the mechanism of injury of his patient because it was obvious. The combination of forces acting across the upper limb in different directions and levels of energy makes that the real mechanism in the other three cases, including our patient could not be known.

Several treatment modalities have been reported for the floating elbow, mainly related to the humerus, including: closed reduction and cast, open reduction and internal fixation, intramedullary nailing, and external fixation. ${ }^{1-3,5,6}$ Rogers et $\mathrm{al}^{3}{ }^{3}$ reported $100 \%$ nonunion rate of humeral fractures treated nonsurgically. Yokoyama et al. ${ }^{6}$ recommended stable internal fixation after reviewing 14 cases. Simpson and Jupiter $^{4}$ stated in their review article that when the floating elbow affects the joint, open reduction, and internal fixation is advisable.

Treatment of the reported case included early stable internal fixation of the forearm fractures, but delayed definitive fixation and bone graft of the humeral fracture due to the soft tissues injuries. Our patient recovered a functional range of motion despite the loss of extension as a result of the narrowed olecranon groove by the bone graft.

After reviewing the literature a classification is suggested, which includes all patterns of injuries reported about floating dislocated elbow. This classification includes three types of injuries organized in order of increasing severity, according to the morphologic complexity:

Type I: Floating dislocated elbow without articular bone injury. 3,8

Type II: Floating dislocated elbow with distal radioulnar joint dislocation. ${ }^{7}$

Type III: Floating dislocated elbow with articular fracture of the distal aspect of the humerus (present case).

We have presented a variant of floating dislocated elbow with articular disruption of the distal humerus, which was treated with anatomic reduction, stable, and rigid internal fixation. This treatment allowed early rehabilitation restoring a functional range of motion.

\section{REFERENCES}

1. Capomassi M, Slullitel M. Codo flotante del adulto. Rev Asoc Arg Ortop y Traumatol. 2001;66:99-105.

2. Lange R, Foster R. Skeletal management of shaft fractures associated with forearm fractures. Clin Orthop. 1980;153:218-222.

3. Rogers J, Bennett J, Tullos H. Management of concomitant ipsilateral fractures of the humerus and forearm. J Bone Joint Surg. 1984;66A:552-556.

4. Simpson NS, Jupiter JB. Complex fracture patterns of the upper extremity. Clin Orthop. 1995;318:43-53.

5. Solomon HB, Zadnik M, Eglseder A. A review of outcomes in 18 patients with floating elbow. J Orthop Trauma. 2003;17:563-570.

6. Yokoyama K, Itoman M, Kobayashi A, Shindo M, Futami T. Functional outcomes of "floating elbow" injuries in adults patients. J Orthop Trauma. 1998;12:284-290.

7. Sarup S, Bryant P. Ipsilateral humeral shaft and Galeazzi fractures with posterolateral dislocation of the elbow: a variant of the "Floating Dislocated Elbow." J Trauma. 1997;43:349-352.

8. Viegas SF, Gogan W, Riley S. Floating dislocated elbow: A case report and review of the Literature. J Trauma. 1989;29:886-888. 